\title{
Fusion of HSV-1 VP22 to a bifunctional chimeric SuperCD suicide gene compensates for low suicide gene transduction efficiencies
}

\author{
MARIE-LUISE LEMKEN*, FLORIAN GRAEPLER*, CLAUDIA WOLF, WOLFGANG A. WYBRANIETZ, \\ IRINA SMIRNOW, ULRIKE SCHMIDT, MICHAEL GREGOR, MICHAEL BITZER and ULRICH M. LAUER
}

Department of Internal Medicine I, University Clinic Tübingen, D-72076 Tübingen, Germany

Received December 18, 2006; Accepted February 8, 2007

\begin{abstract}
Low transduction efficiencies of viral and non-viral vectors still remain a major limitation in suicide gene therapy. The HSV-1 tegument protein VP22 can spread from cells where it is produced to surrounding recipient cells, thus making it a promising tool for compensation of inadequate gene transfer efficiencies. In our previous study, we focused on the optimization of the cytosine deaminase (CD) suicide gene system for the treatment of hepatocellular carcinoma. The fusion of yeast cytosine deaminase (YCD) to yeast uracilphosphoribosyltransferase designated SuperCD was shown to be catalytically superior to the YCD gene in our previous study. The aim of our study was to investigate whether fusion of the bifunctional SuperCD suicide gene to VP22 could further potentiate suicide gene therapy efficiency. C- and Nterminal fusions of SuperCD linked in-frame with VP22 were created and cloned into recombinant adenoviral vectors. Under incubation with the prodrug 5-fluorocytosine (5-FC) a strong enhancement in suicide gene induced target cell cytotoxicity was observed whereby the C-terminal fusion of VP22 to SuperCD (VP22-SuperCD) caused the most tremendous decrease in $\mathrm{IC}_{50}$ compared to both Ad-SuperCD transduced and uninfected hepatoma control cells. Optimization of the bystander effect mediated by the intercellular transport of VP22-fusion proteins was demonstrated by cytotoxicity assays performed with a mixture of adenoviral transduced cells and naïve uninfected cells. Immunofluorescence analysis of adenoviral transduced COS- 1 cells coplated with naïve HeLa cells further confirmed the unique property of VP22 for intercellular trafficking.
\end{abstract}

Correspondence to: Dr Ulrich M. Lauer, Department of Internal Medicine I, Medical University Clinic Tübingen, Otfried-MüllerStr. 10, D-72076 Tübingen, Germany

E-mail: ulrich.lauer@uni-tuebingen.de

${ }^{*}$ Contributed equally

Key words: HSV-1, suicide gene, VP22, bystander effect

\section{Introduction}

Suicide gene therapy offers a new promising strategy in the treatment of solid tumors (1). In this concept, a gene of nonhuman origin is transferred to cancer cells by viral or nonviral gene transfer systems rendering target cells specifically sensitive to a so-called prodrug that is relatively non-toxic to normal tissues (2).

However, suicide gene therapy protocols using viral or non-viral vectors are still limited by the inability to transduce the entire tumor cell population $(3,4)$. One strategy to overcome this problem is to substantially improve the so-called bystandereffect, which is defined as the ability of suicide gene transduced cells to mediate cytotoxicity of neighbouring untransduced cells $(5,6)$. The HSV-1 tegument protein VP22 (7) exhibits the remarkable property of intercellular trafficking from the cell where it is produced to many surrounding cells (8) and has been successfully applied in different gene therapeutic approaches, e.g. reporting an increase in cell death when employing VP22-Tk $(9,10)$ or VP22-CD $(11,12)$ fusion genes instead of unfused thymidine kinase (Tk) and bacterial cytosine deaminase (BCD) suicide genes.

The SuperCD suicide gene consists of a fusion between the catalytically superior yeast cytosine deaminase (YCD) (13) gene and the yeast uracil phosphoribosyltransferase gene (UPRT) (14) and was shown to exhibit a stronger suicide gene effect compared to BCD and YCD solely both in vitro as well as in a rat hepatoma model in vivo in our previous study (15).

The aim of our study was to investigate whether fusion of the bifunctional SuperCD suicide gene to VP22 could further potentiate suicide gene therapy efficiency. For this purpose, we generated adenoviral vectors encoding $\mathrm{N}$ - and $\mathrm{C}$-terminal fusions of the optimized suicide gene SuperCD (15) and the HSV-1 tegument protein VP22 (7) and investigated the property of these new vectors to enhance bystander cell killing mediated by the intercellular transport of VP22/SuperCD fusion proteins.

\section{Materials and methods}

Cells. The human hepatoma cell line HepG2 was purchased from the European Collection of Cell Cultures (ECACC, Porton 
Down, Salisbury, Wiltshire, UK), human embryo kidney derived HEK293 cells were purchased from Microbix Biosystems Inc., Ontario, Canada. The cell line HeLa (human cervix carcinoma) was purchased from the American Type Culture Collection (ATCC, Rockeville, MD, USA). 293-E4 5/38 cells were a kind gift from Transgene SA (Strasbourg, France). COS-1 (monkey kidney) cells were from A. Phelan, Marie Curie Research Institute, Oxted, UK. MH (Morris hepatoma) 3924A cells were purchased from the German Cancer Research Center (DKFZ) Tumor Collection, Heidelberg, Germany. All cells were maintained in Dulbecco's modified Eagle's medium (DMEM; Cambrex, East Rutherford, NJ, USA) with $10 \%$ fetal bovine serum and cultivated in a humidified incubator at $37^{\circ} \mathrm{C}$ in a $5 \% \mathrm{CO}_{2}$ atmosphere.

Plasmid constructions. Adenoviral plasmid pTG6600-SuperCD was generated as described previously (15). For generation of pTG6600-SuperCD-VP22, SuperCD was amplified from plasmid pTG6600-SuperCD with primers 5'-TTC GCT AGC GCC ACC ATG GTG ACA GGG GGA ATG-3' (NheIYCD_for) and 5'-TTC GAA TTC AAC ACA GTA GTA TCT GTC ACC AAA GTC ACC-3' (EcoRI-YUPRT_rev), enzymatically digested with NheI and EcoRI and inserted into the equivalently cut vector pTG6600, generating plasmid pTG6600-SuperCDII. Next, VP22 was amplified from ptkVP22ep (a kind gift from P. O'Hare, Marie Curie Research Institute, Oxted, UK) with primers 5'-TTC GAA TTC ATG ACC TCT CGC CGC TCC-3' (EcoRI-VP22_for) and 5'-TTC GCGG CCGC TTA CTC GAC GGG CCG TCT-3' (NotIVP22_rev) and subcloned into the EcoRI/NotI cut vector pTG6600-SuperCDII, resulting in generation of plasmid pTG6600-SuperCD-VP22.

PCR with primers 5'-TTC GAA TTC AGC CCA AGC TCA AAA GAT C-3' for (EcoRI-VP22_for) and 5'-TTG GAT ATC CTC GAC GGG CCG TCT GGG G-3' rev (EcoRVVP22_rev) and plasmid ptk-VP22ep as template and subsequent cloning of the $E c o \mathrm{RI} / E c o \mathrm{RV}$ digested fragment into the equivalently cut vector pUC29 gave rise to plasmid pUC-VP22. pUC-SuperCD served as template for generation of an EcoRVSuperCD-NotI fragment using primers 5'-TTG GAT ATC GCC ACC ATG GTG ACA GGG GG-3' for (EcoRV-SuperCD_for) and 5'-TCC GCG GCC GCC TTA AAC ACA GTA G-3'_rev (NotI-SuperCD'rev). This fragment was cloned into the EcoRV/NotI digested plasmid pUC-VP22, thereby generating plasmid pUC-VP22-SuperCD. Finally, for generation of adenoviral plasmid pTG6600-VP22-SuperCD, VP22-SuperCD was excised from pUC-VP22-SuperCD by digestion with EcoRI and Not I and cloned into the equivalently cut vector pTG6600. pVP22GFP was generated by inserting the BglII/BamHI 911-bp fragment of ptk-VP22ep, containing the VP22 coding region, into the equivalently cut vector pEGFP-N1 (Clontech, Heidelberg, Germany). All constructs were sequenced thereby confirming their predicted composition.

Virus generation, purification and titration. Homologous recombination of the respective adenoviral shuttle plasmids pTG6600-SuperCD, pTG6600-SuperCD-VP22, and pTG6600VP22-SuperCD with plasmid pTG15083 (16) into E. coli BJ5183 resulted in generation of infective adenoviral vectors Ad-SuperCD, Ad-SuperCD-VP22, and Ad-VP22-SuperCD.
Homologous recombination of the respective adenoviral shuttle plasmid pVP22GFP with plasmid AdEasy-1 (17) into E. coli BJ5183 resulted in generation of infective adenoviral vector Ad-VP22GFP. Adenoviral vector Ad-0, containing no foreign transgene cassette, served as a control. Viral genomes were released from the respective recombinant plasmids by $P a c I$ digestion and transfected into the 293 complementation cell line as described previously (16). As pTG15083 contains an additional deletion in the viral E4 region (ORF 3/ORF 4) (18), 293-E4 5/38 packaging cells were used for the generation of Ad-0, Ad-SuperCD, Ad-SuperCD-VP22 and Ad-VP22SuperCD. Transfections were performed using the multicomponent lipid-based FuGENE $6^{\mathrm{TM}}$ transfection reagent (Roche Diagnostics, Mannheim, Germany) according to the manufacturer's protocol using $2 \mu \mathrm{g}$ of linearised plasmid DNA. Recombinant viruses were purified using two rounds of $\mathrm{CsCl}$ density gradient centrifugation. Titers were determined by standard plaque assay (19). Using the method of Zhang et al (20) all purified viral preparations were routinely screened to exclude occurrence of replication-competent adenoviruses. Virus preparations were stored at $-80^{\circ} \mathrm{C}$ in $25 \%$ glycerol, $10 \mathrm{mM}$ Tris/ $\mathrm{HCl}$ and $1 \mathrm{mM} \mathrm{MgCl}_{2}$.

SRB cytotoxicity assay. MH3924A rat hepatoma cells and HepG2 human hepatoma cells, respectively, were seeded in $24-w e l l$ plates at a densitiy of $1 \times 10^{4}$ cells/well. The next day cells were transduced with adenoviral vector preparations in serum-reduced OPTI-MEM medium (Life Technologies, Eggenstein, Germany) at different MOI for $1 \mathrm{~h}$ at $37^{\circ} \mathrm{C}$. The next day, prodrug-containing medium was added at concentrations of $0,0.0001,0.001,0.01,0.1$ and $1 \mathrm{mM} \mathrm{5-FC}$ (a generous gift from Roche, Basel, Switzerland). Cells were incubated for 5 days with a change of medium every other day. Then, growth inhibition was evaluated by the SRB assay according to Skehan et al (21). Cells were fixed with $10 \%$ $(\mathrm{w} / \mathrm{v})$ TCA and stained with $0.4 \%(\mathrm{w} / \mathrm{v}) \mathrm{SRB}$ in $1 \%$ acetic acid. After solubilisation with $10 \mathrm{mM}$ Tris $\mathrm{pH}$ 10.5, stained solutions were transferred to a 96-well plate for measurement in a microtiter plate reader (Dynatech MR7000, Denkendorf, Germany) using a 550-nm filter.

Evaluation of bystander effect. For coplating SRB assays, MH3924A or HepG2 cells were transduced with adenoviral vector preparations at MOI 50. The next day, transduced cells were washed extensively with PBS to remove any unattached virus and then mixed with naïve uninfected cells at a ratio of 1:20 and seeded in 24-well plates at a final density of $1 \times 10^{4}$ cells/well. Twenty-four hours later prodrug treatment started and SRB assays were performed as described above.

Coplating assay. To visualize VP22-mediated spread after adenoviral transduction COS-1 target cells were seeded out in 6-well plates at a density of $2.5 \times 10^{5}$ cells/well. The following day adenoviral vector preparations, diluted in serum-reduced Opti-MEM medium (Life Technologies) were added at MOI 100. The transduction was carried out for $1 \mathrm{~h}$ at $37^{\circ} \mathrm{C}$, then the virus-containing medium was aspirated and cells were cultured in fresh medium. The next day VP22-producing transduced cells were washed extensively with PBS to remove any unattached virus, trypsinized, mixed with naïve uninfected 
HeLa recipient cells at a ratio of 1:10 and plated in 6-well plates, each well containing one coverslip. Twenty-four hours later immunofluorescence staining was performed as described below.

Immunfluorescence microscopy. Cells to be processed for immunofluorescence were washed with PBS and fixed with $4 \%$ paraformaldehyde for $20 \mathrm{~min}$ at room temperature. The samples were then blocked with $10 \%$ newborn calf serum in PBS for $15 \mathrm{~min}$ at room temperature. Primary antibody was added in block solution and incubated for $20 \mathrm{~min}$. Following two washes in PBS, the samples were incubated with secondary antibody for $10 \mathrm{~min}$. After two more washes in PBS the coverslips were mounted in a Mowiol 40-88 (Sigma, Deisenhofen, Germany) preparation containing 2.5\% DABCO as an antifade reagent. Cells were visualized employing an Axiovert 135 microscope (Zeiss, Göttingen, Germany) and appropriate filter sets (objective x40, 1.3 NA). All images were captured using a PCO CCD imaging SensiCam 370 XL (Klughammer, Markt Indersdorf, Germany) and the Carl Zeiss Vision AxioVision 3.1 software.

Antibodies. For double staining in immunofluorescence mouse monoclonal anti-SV40 large T antigen antibody (Santa Cruz Biotechnology, Heidelberg, Germany) was used at a 1:200 dilution, polyclonal rabbit antiserum directed against HSV-1 VP22 (AGV30) (8) was used at a dilution of 1:500. Secondary fluorescent antibodies for microscopy were FITC-conjugated anti-mouse IgG (Vector, Burlingame, CA, USA) and Alexa 546 goat anti-rabbit antibody (Molecular Probes, Eugene, OR, USA) both employed at a 1:1.000 dilution.

Statistical analysis. Statistical analysis was performed using JMP IN 5.1 software (SAS Institute Inc., Cary, NC, USA, 2003). For calculation of $\mathrm{IC}_{50}$ values, an inverted E-max model using the least squares method was used:

$$
O D_{i j}=\operatorname{Emax}_{j}\left(1-\frac{C_{i j}}{C_{i j}+E C 50_{j}}\right)+\varepsilon_{i j}, \varepsilon_{i j} \sim N\left(0, \sigma^{2}\right)
$$

Differences between the treatment groups in the coplating SRB assay were determined using Student's t-test. A $p<0.05$ was considered statistically significant.

\section{Results}

Construction and expression analysis of adenoviral vectors encoding VP22/SuperCD fusion genes. To investigate the ability of HSV-1 VP22 to enhance SuperCD/5-FC suicide gene therapy of hepatocellular carcinoma by intercellular protein trafficking we generated adenoviral expression vectors for full-length, in-frame $\mathrm{N}$ - and C-terminal fusion genes of VP22 to the bifunctional yeast cytosine deaminase (YCD)/ yeast uracilphosphoribosyltransferase (YUPRT) fusion gene SuperCD, designated Ad-SuperCD-VP22 and Ad-VP22SuperCD (Fig. 1). Western blot analysis of Morris hepatoma 3924A (MH) and human hepatoma HepG2 cells transduced with adenoviral vectors Ad-SuperCD-VP22 and Ad-VP22SuperCD at MOI 50 confirmed generation of predominantly full-length fusion proteins of the expected size of $75 \mathrm{kDa}$ (data not shown). Also the unfused SuperCD suicide protein

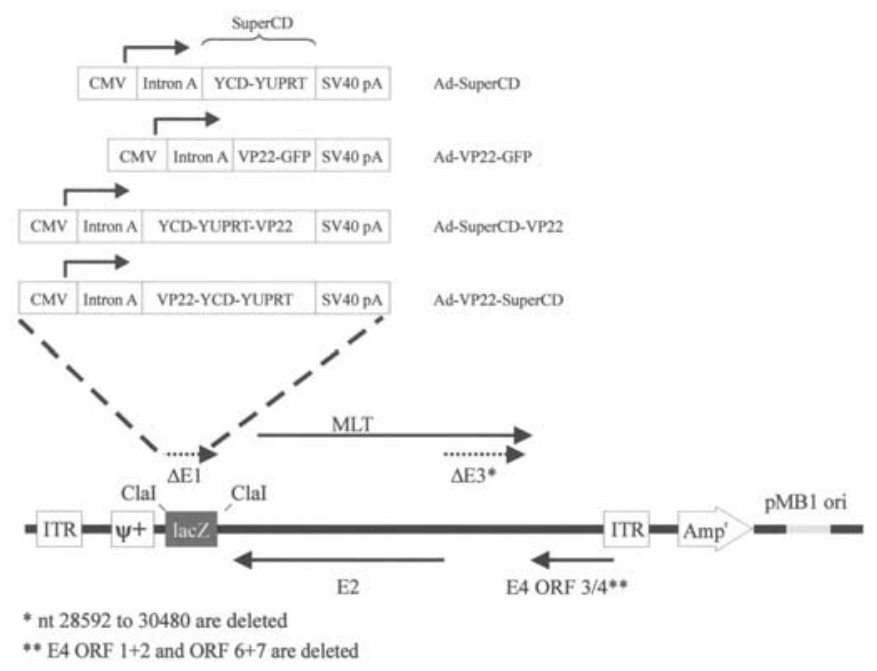

Figure 1. Schematic representation of adenoviral vectors. Adenoviral vectors constructed for this study; CMV, immediate-early enhancer/promoter fragment of the human cytomegalovirus; E, early region; ITR, inverted terminal repeats; $\Psi$, Ad packaging signals; MLT, major late transcription unit; intron A, chimaeric intron of human betaglobin splice donor and mouse IgG splice acceptor sites; SuperCD, fusion protein of yeast cytosine deaminase (YCD) and yeast uracilphosphoribosyltransferase (YUPRT); SV40-pA, simian virus 40 late polyadenylation site; GFP, enhanced green fluorescent protein; VP22, virion protein 22 of herpes simplex virus.

was detected at the expected size of $42 \mathrm{kDa}$ in both $\mathrm{MH}$ as well as in HepG2 cells transduced with adenoviral vector AdSuperCD at MOI 50 (data not shown).

Suicide gene cytotoxicity of VP22/SuperCD fusion genes. To test whether VP22/SuperCD fusions are able to enhance the suicide gene cytotoxic effect of the parental SuperCD gene SRB cytotoxicity assays were performed, which are known as the standard assay of the US National Cancer Institute (NCI) to screen for all kinds of cytotoxic substances (21). $\mathrm{MH}$ and HepG2 cells, respectively, were transduced with adenoviral vectors at different MOI (MOI 50, MOI 10, MOI 5). After 5 days of incubation with the prodrug 5-fluorocytosine (5-FC) growth inhibition was determined by staining with the protein dye sulforhodamin B (SRB). Untransduced cells, as well as control vector Ad-0 transduced cells, being cultured for 5 days with the analogous amounts of 5-FC, were used as controls. SRB values were referred to that of adenoviral transduced cells incubated for 5 days without prodrug incubation and are given as percentage of surviving cells (control, 100\%).

As expected, in both cell lines no significant inhibition of cellular growth under incubation with increasing amounts of 5-FC was detected for naïve/untransduced cells as well as for control vector (Ad-0) infected cells, in both cell lines and for all MOI investigated (Fig. 2).

In contrast, expression of the unfused SuperCD suicide gene resulted in a pronounced cytotoxic effect with increasing amounts of the prodrug 5-FC for all MOI investigated. Infection of the target cells with Ad-SuperCD, Ad-SuperCD-VP22 and Ad-VP22-SuperCD increased the sensitivity of the cells to 5 -FC in a virus dose-dependent manner.

However, when comparing the inhibitory effect of AdSuperCD with Ad-SuperCD-VP22 and Ad-VP22-SuperCD, an enhanced suicide gene effect of the VP22 fusion proteins 
MH

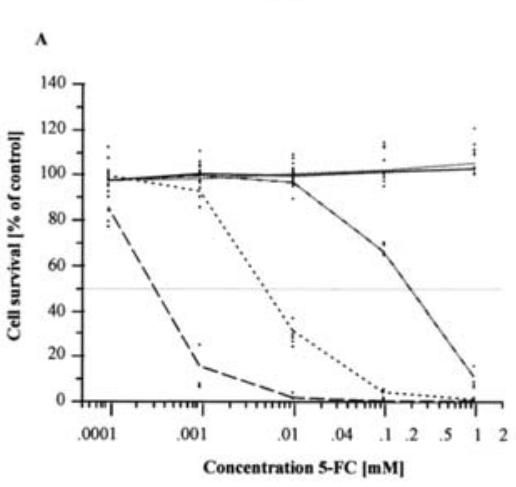

c

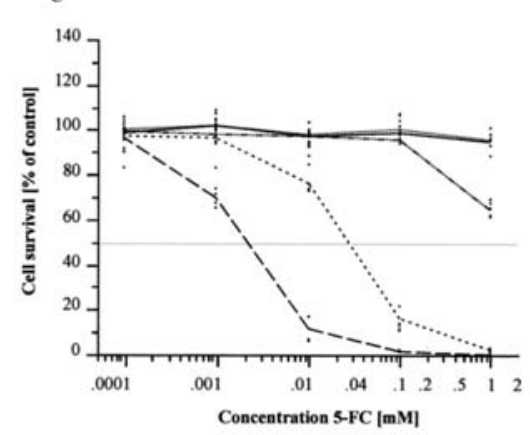

E

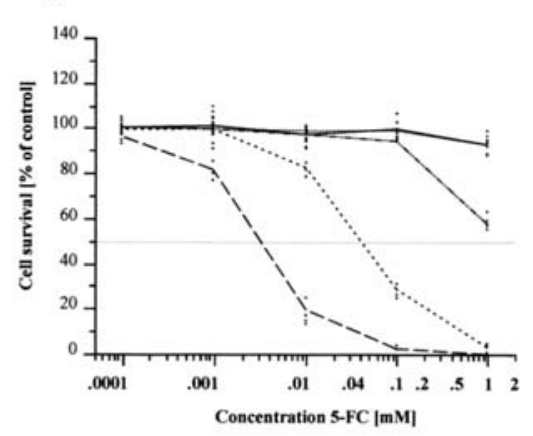

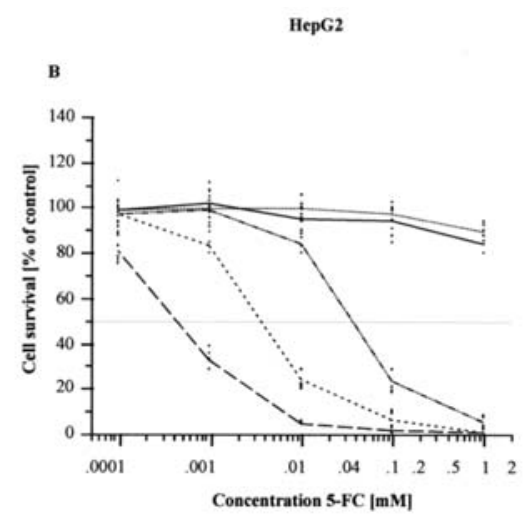

D

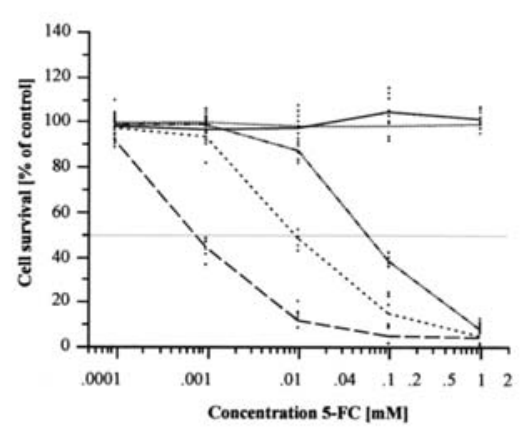

$\mathbf{F}$

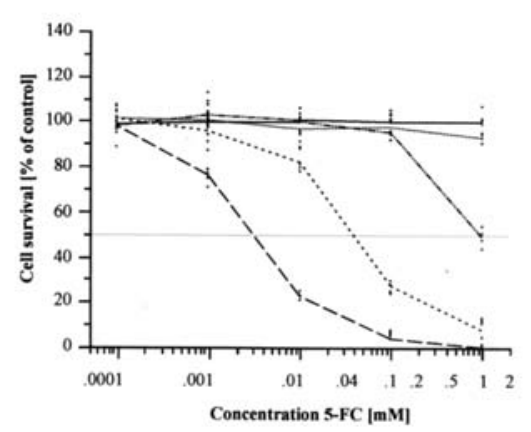

- untransduced control cells
Ad-0 transduced control cells Ad-0 transduced control cells

..... Ad-SuperCD-VP22 transduced cells -- Ad-VP22-SuperCD transduced cells

Figure 2. SRB cytotoxicity assay with recombinant adenoviral vectors. Rat Morris hepatoma cells MH3924A (A, C snd E) or human hepatoma cells HepG2 (B, D and F) were transduced with adenoviral vectors Ad-0, Ad-SuperCD, Ad-SuperCD-VP22 and Ad-VP22-SuperCD (MOI 50, MOI 10, MOI 5). After 5 days of prodrug incubation at the concentrations given growth inhibition was determined by SRB staining and measured at $550 \mathrm{~nm}$. All values refer to those of the control cells (adenoviral transduced cells without prodrug incubation) and are depicted as the percentage of surviving cells (control, 100\%). All experiments were carried out in quadruplicate and repeated once.

was found at every distinct 5-FC concentration level. The concentration of 5-FC necessary to accomplish a 50\% inhibition of cellular growth (inhibitory concentration $\mathrm{IC}_{50}$ ) was found to be strongly reduced in both Ad-SuperCD-VP22 and Ad-VP22-SuperCD infected cells compared to Ad-SuperCD infected cells for all MOI investigated (Table I). When comparing Ad-SuperCD infected cells and Ad-SuperCD-VP22 infected cells, $\mathrm{IC}_{50}$ was reduced between 23 -fold and 37-fold in $\mathrm{MH}$ cells and between 6-fold and 25-fold in HepG2 cells at the different MOI investigated (Table I, column III vs. column IV). Moreover, transduction with Ad-VP22-SuperCD even achieved a much stronger enhancement in suicide gene killing compared to Ad-SuperCD infected cells (Table I, column III vs. column V: reduction of $\mathrm{IC}_{50}$ between 305 - and 667-fold in MH cells and between 75- and 317-fold in
HepG2 cells). HepG2 cells infected with Ad-SuperCD were found to be very sensitive to 5-FC treatment at MOI 50 and MOI 10 (Fig. 2 and Table I), therefore the highest difference in $\mathrm{IC}_{50}$ between Ad-SuperCD-VP22/Ad-VP22-SuperCD and Ad-SuperCD transduced cells was achieved at MOI 5.

At a 5-FC concentration of only $0.01 \mathrm{mM}$, even at MOI 5, only $20 \%$ of the Ad-VP22-SuperCD infected cells were found to survive in both cell lines; in sharp contrast, survival of Ad-SuperCD transduced MH cells amounts to $>90 \%$ at this 5-FC concentration, even when a high MOI (MOI 50) was employed. Comparable results were found for Ad-SuperCD transduced HepG2 cells.

The cytotoxic effect caused by adenoviral transduction of the target cells not incubated with 5-FC was found to be $<20 \%$ for both MH and HepG2 cells at MOI 10 and MOI 5 
Table I. $\mathrm{IC}_{50}$ of 5-FC inhibition of cellular growth (as determined by SRB assays).

\begin{tabular}{llccc}
\hline Cell type & Treatment & $\begin{array}{c}\text { Ad-SuperCD IC } \\
{[\mathrm{mM} 5-\mathrm{FC}]}\end{array}$ & $\begin{array}{c}\text { Ad-SuperCD-VP22 } \text { IC }_{50} \\
{[\mathrm{mM} \mathrm{5-FC}]}\end{array}$ & $\begin{array}{c}\text { Ad-VP22-SuperCD IC } \\
{[\mathrm{mM} 5 \text { 5-FC }]}\end{array}$ \\
\hline MH & MOI 50 & 0.2 & 0.006 & 0.0003 \\
& MOI 10 & $>1$ & 0.03 & 0.002 \\
& MOI 5 & $>1$ & 0.04 & 0.003 \\
& Coplating & & 0.03 \\
HepG2 & $>1$ & 0.4 & 0.0005 \\
& MOI 50 & 0.04 & 0.004 & 0.001 \\
& MOI 10 & 0.07 & 0.01 & 0.003 \\
& MOI 5 & 1.0 & 0.04 & 0.07 \\
\hline
\end{tabular}

${ }^{\mathrm{a}}$ Mixture of 5\% adenoviral transduced cells and 95\% non-transduced naïve cells (SRB assay described in Fig. 4).

MH

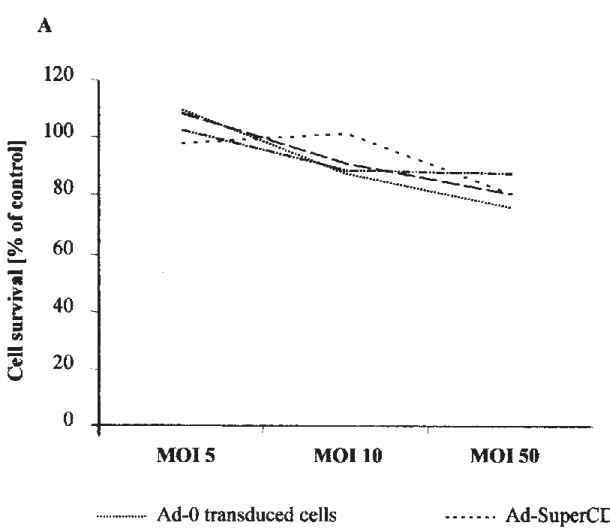

HepG2

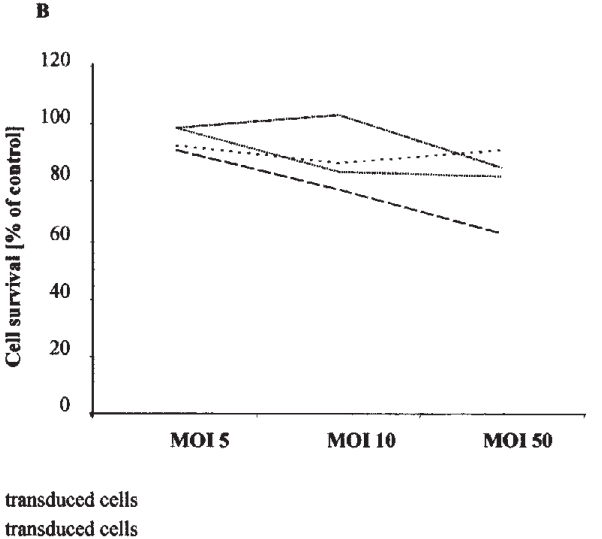

Figure 3. Determination of adenoviral vector toxicity. OD values of cells transduced with adenoviral vectors Ad-0, Ad-SuperCD, Ad-SuperCD-VP22, and Ad-VP22-SuperCD at different MOI (MOI 50, MOI 10, MOI 5) refer to those of naïve cells. Cells were treated as described in Fig. 2, but without any prodrug incubation.

(Fig. 3); at MOI 50, a slightly stronger effect was observed in HepG2 cells transduced with Ad-VP22-SuperCD (Fig. 3B). These control data are in strong support of the notion that the clearly enhanced suicide gene effect achieved by employment of our new VP22/SuperCD adenoviral vector fusions cannot be explained by any unspecific transgene induced cytotoxicity.

VP22 mediated enhanced bystander cell killing. To further investigate whether the improved cytotoxic effect of the VP22/SuperCD fusion proteins is caused by an enhanced transduction efficency due to VP22-mediated intercellular transport of the fusion proteins to neighbouring non-transduced cells, SRB cytotoxicity assays were performed with a mixture of adenoviral transduced and non-transduced naïve cells. Cells were transduced with adenoviral vectors Ad-0, AdSuperCD, Ad-SuperCD-VP22, and Ad-VP22-SuperCD at MOI 50. The next day transduced cells were mixed with naïve uninfected cells at a ratio of 1:20. After 5 days of 5-FC prodrug treatment
SRB staining was performed as described above. As expected, both uninfected cells and Ad-0 infected control cells showed $100 \%$ viability (Fig. 4). Under the chosen conditions (5\% infected cells) no significant cytotoxic effect could be achieved employing parental adenoviral vector Ad-SuperCD, even at the highest prodrug concentration investigated. In contrast, differences between Ad-SuperCD-VP22 and control cells were significant at a prodrug concentration of $1 \mathrm{mM}$ in HepG2 cells $(\mathrm{p}<0.001)$ and at a concentration of $0.1 \mathrm{mM} \mathrm{5-FC}$ and $1 \mathrm{mM} 5$-FC in MH cells $(\mathrm{p}<0.001)$. For Ad-VP22-SuperCD, differences were significant at a concentration of $0.1 \mathrm{mM}$ and $1 \mathrm{mM} 5-\mathrm{FC}$ in HepG2 cells $(\mathrm{p}<0.001)$ and at a concentration of $0.001 \mathrm{mM} 5-\mathrm{FC}(\mathrm{p}<0.05)$ as well as at 5-FC concentrations of $0.01,0.1$ and $1 \mathrm{mM} \mathrm{5-FC}(\mathrm{p}<0.001)$ in $\mathrm{MH}$ cells. $\mathrm{IC}_{50}$ values for Ad-SuperCD-VP22 were $0.4 \mathrm{mM} 5-\mathrm{FC}(\mathrm{MH})$ and $0.5 \mathrm{mM}$ 5-FC (HepG2). $\mathrm{IC}_{50}$ values for Ad-VP22-SuperCD were $0.03 \mathrm{mM} 5$-FC in $\mathrm{MH}$ and $0.07 \mathrm{mM} 5$-FC in HepG2 (Table I). These results impressively point to a strong additional 
MH

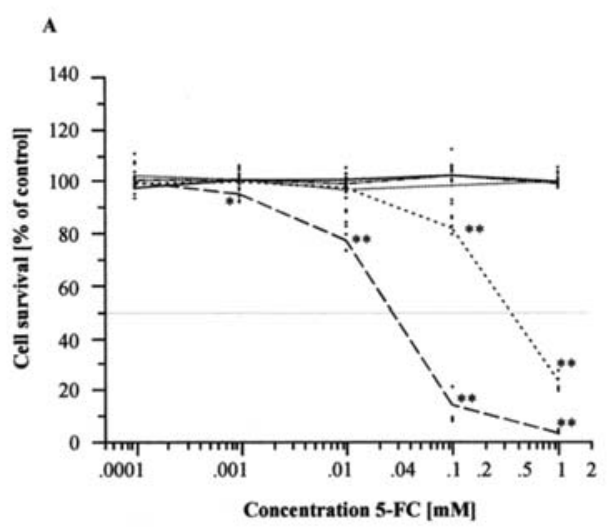

HepG2

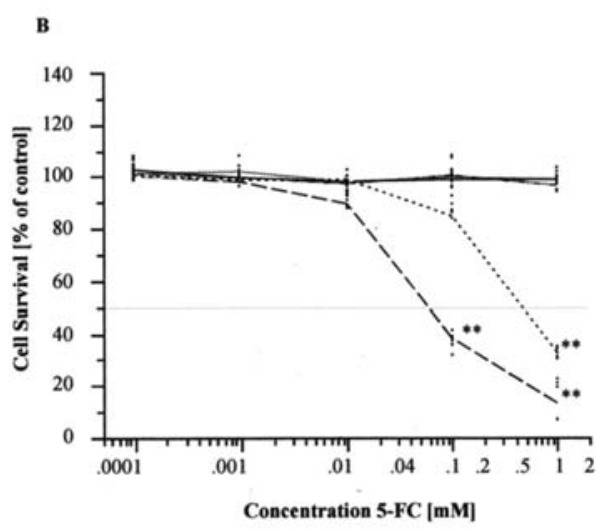

\section{\begin{tabular}{lllll} 
- untransduced control cells & ....... Ad-SuperCD-VP22 transduced cells \\
\hline Ad-0 transduced control cells & -
\end{tabular} \\ Adransduced control cells}

Figure 4. Analysis of VP22 mediated enhanced bystander cell killing. Hepatoma cells were transduced with adenoviral vectors at MOI 50. The next day transduced cells were mixed with naïve uninfected cells at a ratio of 1:20. After 5 days of prodrug incubation at the concentrations given SRB staining was performed as described for Fig. 2. All values refer to those of the control cells (adenoviral transduced, but without prodrug incubation) and are given as percentage of surviving cells (control, $100 \%$ ). All experiments were carried out at least in quadruplicate and repeated once. ${ }^{*} \mathrm{p}<0.05 ;{ }^{* *} \mathrm{p}<0.001$.
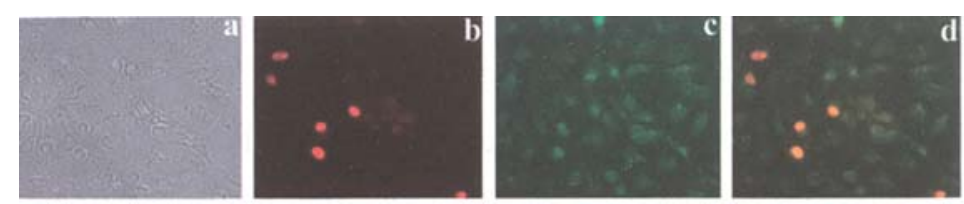

mock
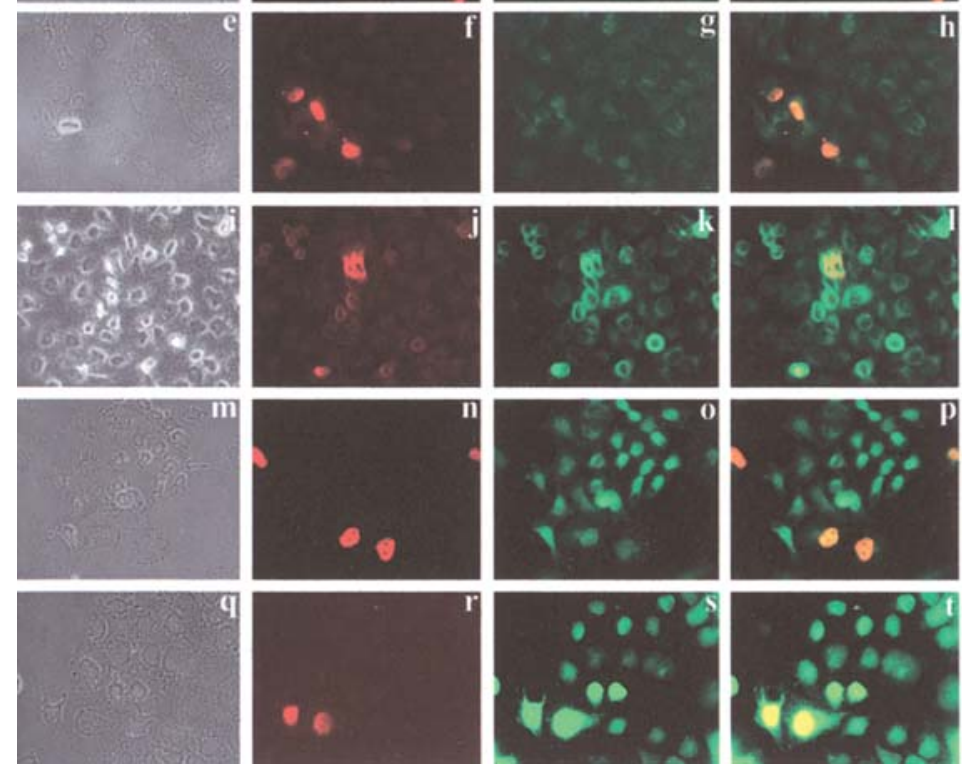

Ad-0

Ad-SuperCD-VP22

Ad-VP22-SuperCD

Ad-VP22-GFP

Figure 5. Coplating of COS-1 and HeLa cells after transduction with adenoviral vectors. COS-1 cells were transduced with adenoviral vectors at MOI 50 . Twenty-four hours later cells were trypsinized and coplated on to preseeded HeLa cells at a ratio of 1:10. Immunofluorescence detection was carried out twenty-four hours later. All coplatings were stained with two primary antibodies, anti-SV40 T antigen to identify the COS-1 cells in all samples (second antibody Alexa 546 conjugated; left column) plus anti-VP22 antibody (second antibody FITC-conjugated; middle column) to detect VP22-mediated intercellular trafficking; left column, transmitted light images; right column, merge display. Upper row, uninfected cells (control); second row, transduction with Ad-0 (negative vector control); third row, transduction with Ad-SuperCD-VP22; fourth row, transduction with Ad-VP22-SuperCD; fifth row, transduction with Ad-VP22-GFP.

bystander effect induced by intercellular protein transport of the VP22/SuperCD fusion proteins.

Intercellular transport of VP22/SuperCD fusion proteins. To visualize the intercellular transport property of VP22/SuperCD fusion proteins, indirect immunofluorescence microscopy analysis of coplated mixtures of adenoviral transduced cells and naïve recipient cells was performed (Fig. 5). As monkey kidney COS-1 cells constitutively express the SV40 large T antigen, coplating of adenoviral transduced COS-1 cells with SV40 large T antigen negative naïve cells of another cell type, e.g. HeLa cells, facilitates distinction of the export 
of VP22 fusion proteins from the adenovirus-transduced producer cells into neighbouring untransduced recipient cells by indirect immunofluorescence staining. COS-1 cells were transduced with either adenoviral vector at MOI 50. Then, adenoviral transduced COS-1 cells were washed extensively with PBS to remove any unattached virus, trypsinized and mixed with naïve uninfected HeLa recipient cells at a ratio of 1:10. The next day all coplating cultures were fixed with the mild fixation reagent paraformaldehyde and stained with two primary antibodies: i) anti-SV40 T antigen to identify primary adenoviral transduced COS-1 cells in all samples (Alexa 546 conjugated antibody employed as secondary antibody; second column in Fig. 5b, f, j, n and r) plus an ii) anti-VP22 antibody (FITC-conjugated antibody employed as secondary antibody; third column in Fig. 5c, g, k, o and s) to detect VP22 mediated intercellular trafficking.

As a result, untransduced COS-1 cells mixed with naïve HeLa cells (mock control) clearly show detection of SV40 large $\mathrm{T}$ antigen expressing COS-1 cells (Fig. $5 \mathrm{~b}$ and d), but only slightly green background fluorescence (Fig. 5c, merge overlay in d). Similar results were obtained when COS-1 cells being transduced with adenoviral control vector Ad-0 were mixed with naïve HeLa cells: expression of SV40 large $\mathrm{T}$ antigen in COS-1 cells (Fig. $5 \mathrm{f}$ and $\mathrm{h}$ ) and again minor green background fluorescence (Fig. 5g and merge overlay in $\mathrm{h}$ ).

However, when COS-1 cells being transduced with AdSuperCD-VP22 were mixed with naïve HeLa cells, a typical pattern of VP22-mediated transport is detected (Fig. 5, third row). Primary adenoviral transduced cells expressing both the SV40 large T antigen as well as VP22 can be easily identified by their yellow colour in the merge overlay (Fig. 51). These cells were found to be surrounded by numerous HeLa recipient cells being only green but not red stained due to VP22 intercellular trafficking of the SuperCD-VP22 fusion proteins. When mixing COS-1 cells transduced with Ad-VP22-SuperCD and non-transduced HeLa cells, the same pattern of VP22 intercellular trafficking can be observed (Fig. 5p).

To preclude the possibility that primary and secondary antibodies could have caused background fluorescence artefacts in the detection of VP22 intercellular trafficking (Fig. 5c and g), we directly investigated the trafficking property of VP22 with a VP22-GFP fusion protein (Fig. 5, lower row), thereby circumventing the need for the usage of any VP22 antibodies. As a result, primary transduced COS-1 cells are again surrounded by many HeLa recipient cells exhibiting only green fluorescence due to VP22 intercellular trafficking of the VP22-GFP fusion protein (Fig. 5t). Hence, direct fluorescence microscopy further approves the property of the VP22 fusion proteins for intercellular trafficking.

\section{Discussion}

Suicide gene therapy offers a promising strategy in the treatment of cancer. Numerous pre-clinical and clinical studies employing adenoviral suicide gene therapy have been conducted obtaining encouraging results, but robust clinical efficacy remains elusive (22).

Any successful clinical application of this therapeutic approach depends on the efficiency of tumor cell transduction.
One strategy to improve suicide gene therapy is to enhance the sensitivity of the target cells to the prodrug by the generation of new bifunctional suicide genes $(14,23)$. We previously investigated the cytotoxic effect of the bifunctional SuperCD suicide gene, encoding a fusion of the yeast cytosine deaminase gene with the yeast uracil phosphoribosyltransferase gene (YCD::YUPRT). Our study demonstrated a strongly enhanced suicide gene effect of this fusion gene in vitro as well as in vivo compared to the single application of bacterial cytosine deaminase or the yeast cytosine deaminase gene (15).

Optimization of the so-called bystander effect, the delivery of toxified prodrug from primary transduced to neighbouring non-transduced cells, helps to overcome low primary transduction efficiencies of viral and non-viral vectors (6). The HSV-1 tegument protein VP22 has been shown to exhibit the property of spreading from one primary transduced cell to many surrounding cells, thus making it a promising tool for compensation of inadequate gene transfer efficiencies (24). Fusions to VP22 have been shown to improve the therapeutic efficiency of several suicide genes, e.g. the bacterial cytosine deaminase (11), the yeast cytosine deaminase (12) and the herpes simplex virus type 1 thymidine kinase $(10,25,26)$.

The aim of our study was to investigate whether fusion of VP22 to the bifunctional SuperCD suicide gene could further improve the efficiency of SuperCD suicide gene therapy. Employing SRB cytotoxicity assays we found that adenoviral transduction with Ad-SuperCD-VP22 and Ad-VP22-SuperCD led to a much stronger cytotoxic effect compared to AdSuperCD alone (Fig. 2). A reduction in $\mathrm{IC}_{50}$ of up to $>500$-fold at MOI 50 compared to Ad-SuperCD infected cells was achieved with adenoviral vector Ad-VP22-SuperCD (Table I, $0.2 \mathrm{mM} 5$-FC vs. $0.0003 \mathrm{mM} 5-\mathrm{FC}$ ). We did not detect any significant transgene induced cytotoxicity in the absence of 5-FC; only when HepG2 cells were transduced with adenoviral vector Ad-VP22-SuperCD at MOI 50, a cytotoxic effect independent from prodrug treatment was observed (Fig. 3). Further investigations will be needed to specifiy this effect and to prove whether similar mechanisms as shown for the bovine VP22 protein (BVP22) (27) can be assumed for induction of cytotoxicity at high MOI levels. However, the strongly enhanced suicide gene effect achieved by employment of our new VP22/SuperCD adenoviral vector fusions even at MOI 5 cannot be explained by unspecific transgene induced cytotoxicity.

It has been hypothesized that the enhanced cytotoxic effect achieved by the fusion of a suicide gene to VP22 is only caused by an enhanced intracellular effect $(28,29)$, but not due intercellular trafficking of the fusion protein. To prove this assumption, we performed coplating experiments with a mixture of 5\% adenoviral transduced cells and $95 \%$ non-transduced naïve cells (Fig. 4). At these conditions, transduction with the SuperCD suicide gene did not reveal any cytotoxic effect in hepatoma cells, thereby indicating the limitations of current suicide gene systems at low transduction efficiencies. However, when employing our new fusion genes of VP22 to SuperCD, up to $90 \%$ of cells were killed in a mixture of only $5 \%$ adenoviral transduced cells with $95 \%$ naïve cells (Fig. 4). We thereby confirm, that the enhanced cytotoxic effect obtained by transduction with Ad-VP22SuperCD and Ad-SuperCD-VP22 is caused by an enhanced 
bystander effect due to intercellular trafficking of the fusion proteins.

Despite promising results of gene therapeutic approaches using fusions of VP22 to the therapeutic gene of interest $(11,12,30,31-33)$, there still exists a controversy regarding the point of VP22 intercellular trafficking (29,34-37). It has been stated that some of the published results on spreading phenomena can only be explained by methanol fixation artefacts $(34,36)$. Furthermore, recently published data proclaim that VP22 cannot efficiently translocate between cells at all $(29,36)$.

In a recently published study, we created an adenoviral vector with a dual expression cassette for VP22-GFP and DsRed under the control of distinct human CMV promoters. Application of this vector enabled us to clearly distinguish between primary transduced cells and cells taking up VP22GFP by intercellular trafficking. We demonstrated by live cell confocal fluorescence microscopy that VP22 intercellular trafficking is not an artefact caused by fixation of the cells, but also takes place in living unfixed cells (38). To visualize VP22 intercellular spread of our VP22/SuperCD fusion proteins, we mixed adenoviral transduced cells with naïve, non-transduced cells at a ratio of 1:10 and examined the cells by indirect immunofluorescence analysis (Fig. 5). The results confirm a distinct VP22-mediated intercellular trafficking effect of the VP22/SuperCD fusion proteins and are in accordance with our previous studies on VP22-mediated intercellular trafficking $(11,38,39)$ and the results of our actual coplating SRB cytotoxicity assays (Fig. 4).

Whereas both the N-terminal as well as the C-terminal fusion between SuperCD and VP22 strongly enhanced the suicide gene cytotoxicity of SuperCD alone, the C-terminal fusion VP22-SuperCD was found to be more efficient than the N-terminal fusion SuperCD-VP22 in bystander cell killing (Figs. 2 and 4). So far, most studies examining VP22 fusion protein expression analyzed only one kind of fusion construct $(40,41)$ or did not detect differences in intercellular transport or enhancement of cell killing employing VP22-P53 and VP22-Tk fusion proteins $(9,32)$. In our own previous study we detected enhanced cell killing only by an $\mathrm{N}$-terminal CD-VP22 fusion protein but not with the C-terminal fusion VP22-CD (11), similar observations have also been made with BV22-Etk fusion proteins (28). On the other hand, the first report describing the intercellular trafficking property of a fusion between VP22 and a large polypeptide has been made on the C-terminal fusion protein VP22-GFP (8). The contradictory results employing $\mathrm{C}$ - and $\mathrm{N}$-terminal fusions of VP22 may be explained both by i) different size and ii) protein folding properties of the VP22 fusion partners, masking functional domains in the fusion protein. Further research is needed regarding this point.

In conclusion, employing our new adenoviral vectors encoding a fusion of our enzymatically enhanced suicide gene SuperCD to VP22, we were able to potentiate in vitro suicide gene therapy efficiencies. Regarding possible future in vivo applications, a combination between suicide gene therapy and virotherapy, e.g., the introduction of our new suicide gene fusion VP22-SuperCD into tumor selectively replicating vectors $(42,43)$, might help to enhance the oncolytic effect of such virotherapeutics, thereby improving the efficiency of cancer gene therapy.

\section{Acknowledgements}

We are grateful to Reinhard Vonthein for his excellent assistance in statistical analysis. This study was supported in part by grants from the Deutsche Forschungsgemeinschaft (LA 649/20-1, LA 649/20-2). W.A.W. was supported by a scholarship from the Pinguin Foundation (Henkel KGaA).

\section{References}

1. Dachs GU, Tupper J and Tozer GM: From bench to bedside for gene-directed enzyme prodrug therapy of cancer. Anticancer Drugs 16: 349-359, 2005.

2. Mullen CA, Kilstrup $M$ and Blaese RM: Transfer of the bacterial gene for cytosine deaminase to mammalian cells confers lethal sensitivity to 5-fluorocytosine: a negative selection system. Proc Natl Acad Sci USA 89: 33-37, 1992.

3. Wadhwa PD, Zielske SP, Roth JC, Ballas CB, Bowman JE and Gerson SL: Cancer gene therapy: scientific basis. Annu Rev Med 53: 437-452, 2002.

4. Seth P: Vector-mediated cancer gene therapy: an overview. Cancer Biol Ther 4: 512-517, 2005.

5. Huber BE, Austin EA, Richards CA, Davis ST and Good SS: Metabolism of 5-fluorocytosine to 5-fluorouracil in human colorectal tumor cells transduced with the cytosine deaminase gene: significant antitumor effects when only a small percentage of tumor cells express cytosine deaminase. Proc Natl Acad Sci USA 91: 8302-8306, 1994.

6. Lumniczky K and Safrany G: Cancer gene therapy: combination with radiation therapy and the role of bystander cell killing in the anti-tumor effect. Pathol Oncol Res 12: 118-124, 2006.

7. Elliott GD and Meredith DM: The herpes simplex virus type 1 tegument protein VP22 is encoded by gene UL49. J Gen Virol 73: 723-726, 1992.

8. Elliott G and O'Hare P: Intercellular trafficking and protein delivery by a Herpesvirus structural protein. Cell 88: 223-233, 1997.

9. Dilber MS, Phelan A, Aints A, Mohamed AJ, Elliott G, Smith CI and O'Hare P: Intercellular delivery of thymidine kinase prodrug activating enzyme by the herpes simplex virus protein, VP22. Gene Ther 6: 12-21, 1999.

10. Kong BH, Wang WX, Liu CS, Song L, Ma DX, Qu X, Jiang J, Yang XS, Zhang YZ, Wang B, Wei MQ and Yang QF: Efficacy of Lentivirus-mediated and MUC1 antibody-targeted VP22TK/GCV suicide gene therapy for ovarian cancer. In Vivo 17: 153-156, 2003.

11. Wybranietz WA, Gross CD, Phelan A, O'Hare P, Spiegel M, Graepler F, Bitzer M, Stahler P, Gregor M and Lauer UM: Enhanced suicide gene effect by adenoviral transduction of a VP22-cytosine deaminase (CD) fusion gene. Gene Ther 8: 1654-1664, 2001.

12. Lee KC, Hamstra DA, Bullarayasamudram S, Bhojani MS, Moffat BA, Dornfeld KJ, Ross BD and Rehemtulla A: Fusion of the HSV-1 tegument protein vp22 to cytosine deaminase confers enhanced bystander effect and increased therapeutic benefit. Gene Ther 13: 127-137, 2006.

13. Kievit E, Bershad E, Ng E, Sethna P, Dev I, Lawrence TS and Rehemtulla A: Superiority of yeast over bacterial cytosine deaminase for enzyme/prodrug gene therapy in colon cancer xenografts. Cancer Res 59: 1417-1421, 1999.

14. Erbs P, Regulier E, Kintz J, Leroy P, Poitevin Y, Exinger F, Jund R and Mehtali M: In vivo cancer gene therapy by adenovirusmediated transfer of a bifunctional yeast cytosine deaminase/uracil phosphoribosyltransferase fusion gene. Cancer Res 60: 3813-3822, 2000.

15. Graepler F, Lemken ML, Wybranietz WA, Schmidt U, Smirnow I, Gross CD, Spiegel M, Schenk A, Graf H, Lauer UA, Vontheim R, Gregor M, Armeanu S, Bitzer M and Lauer UM: Bifunctional chimeric SuperCD suicide gene - YCD: YUPRT fusion is highly effective in a rat hepatoma model. World $\mathrm{J}$ Gastroenterol 11: 6910-6919, 2005.

16. Chartier C, Degryse E, Gantzer M, Dieterle A, Pavirani A and Mehtali M: Efficient generation of recombinant adenovirus vectors by homologous recombination in Escherichia coli. J Virol 70: 4805-4810, 1996.

17. He TC, Zhou S, Da Costa LT, Yu J, Kinzler KW and Vogelstein B: A simplified system for generating recombinant adenoviruses. Proc Natl Acad Sci USA 95: 2509-2514, 1998. 
18. Lusky M, Christ M, Rittner K, Dieterle A, Dreyer D, Mourot B, Schultz H, Stoeckel F, Pavirani A and Mehtali M: In vitro and in vivo biology of recombinant adenovirus vectors with E1, E1/E2A, or E1/E4 deleted. J Virol 72: 2022-2032, 1998.

19. Graham FL and Prevec L: Methods for construction of adenovirus vectors. Mol Biotechnol 3: 207-220, 1995.

20. Zhang WW, Koch PE and Roth JA: Detection of wild-type contamination in a recombinant adenoviral preparation by PCR. Biotechniques 18: 444-447, 1995.

21. Skehan P, Storeng R, Monks A, McMahon J, Vistica D, Warren JT, Bokesch H, Kenney S and Boyd MR: New colorimetric cytotoxicity assay for anticancer-drug screening. J Natl Cancer Inst 82: 1107-1112, 1990.

22. Kaplan JM: Adenovirus-based cancer gene therapy. Curr Gene Ther 5: 595-605, 2005.

23. Adachi Y, Tamiya T, Ichikawa T, Terada K, Ono Y, Matsumoto K, Furuta $\mathrm{T}$, Hamada $\mathrm{H}$ and Ohmoto $\mathrm{T}$ : Experimental gene therapy for brain tumors using adenovirusmediated transfer of cytosine deaminase gene and uracil phosphoribosyltransferase gene with 5-fluorocytosine. Hum Gene Ther 11: 77-89, 2000.

24. Elliott G and O'Hare P: Intercellular trafficking of VP22-GFP fusion proteins. Gene Ther 6: 149-151, 1999.

25. Greco O, Joiner MC, Doleh A and Scott SD: VP22-mediated intercellular transport for suicide gene therapy under oxic and hypoxic conditions. Gene Ther 12: 974-979, 2005.

26. Liu CS, Kong B, Xia HH, Ellem KA and Wei MQ: VP22 enhanced intercellular trafficking of HSV thymidine kinase reduced the level of ganciclovir needed to cause suicide cell death. J Gene Med 3: 145-152, 2001

27. Qiu Z, Zhu J, Harms JS, Friedrichsen J and Splitter GA: Bovine Herpesvirus VP22 induces apoptosis in neuroblastoma cells by upregulating the expression ratio of Bax to Bcl-2. Hum Gene Ther 16: 101-108, 2005.

28. Qiu Z, Harms JS, Zhu J and Splitter GA: Bovine herpesvirus tegument protein VP22 enhances thymidine kinase/ganciclovir suicide gene therapy for neuroblastomas compared to herpes simplex virus VP22. J Virol 78: 4224-4233, 2004.

29. Hakkarainen T, Wahlfors T, Merilainen O, Loimas S, Hemminki A and Wahlfors J: VP22 does not significantly enhance enzyme prodrug cancer gene therapy as a part of a VP22-HSVTk-GFP triple fusion construct. J Gene Med 7: 898-907, 2005.

30. Zender L, Kühnel F, Kock R, Manns M and Kubicka S: VP22mediated intercellular transport of p53 in hepatoma cells in vitro and in vivo. Cancer Gene Ther 9: 489-496, 2002.
31. Zavaglia D, Favrot MC, Eymin B, Tenaud C and Coll JL: Intercellular trafficking and enhanced in vivo antitumour activity of a non-virally delivered P27-VP22 fusion protein. Gene Ther 10: 314-325, 2003.

32. Phelan A, Elliott G and O'Hare P: Intercellular delivery of functional $\mathrm{p} 53$ by the herpesvirus protein VP22. Nat Biotechnol 16: 440-443, 1998.

33. Green KL, Southgate TD, Mulryan K, Fairbairn LJ, Stern PL and Gaston K: Diffusible VP22-E2 protein kills bystander cells and offers a route for cervical cancer gene therapy. Hum Gene Ther 17: 147-157, 2006

34. Lundberg M and Johansson M: Is VP22 nuclear homing an artifact? Nat Biotechnol 19: 713-714, 2001

35. Fang B, Xu B, Koch $\mathrm{P}$ and Roth JA: Intercellular trafficking of VP22-GFP fusion proteins is not observed in cultured mammalian cells. Gene Ther 5: 1420-1424, 1998.

36. Roy V, Qiao J, Campos-Lima P and Caruso M: Direct evidence for the absence of intercellular trafficking of VP22 fused to GFP or to the herpes simplex virus thymidine kinase. Gene Ther 12: 169-176, 2004.

37. Zavaglia D, Lin EH, Guidetti M, Pluquet O, Hainaut P, Favrot MC and Coll JL: Poor intercellular transport and absence of enhanced antiproliferative activity after non-viral gene transfer of VP22$\mathrm{P} 53$ or P53-VP22 fusions into 553 null cell lines in vitro or in vivo. J Gene Med 7: 936-944, 2005.

38. Lemken ML, Wolf C, Wybranietz WA, Schmidt U, Smirnow I, Bühring HJ, Mack AF, Lauer UM and Bitzer M: Evidence for the presence of intercellular trafficking of VP22 in living cells. Mol Ther 15: 310-319, 2007.

39. Wybranietz WA, Prinz F, Spiegel M, Schenk A, Bitzer M, Gregor M and Lauer UM: Quantification of VP22-GFP spread by direct fluorescence in 15 commonly used cell lines. J Gene Med 1: 265-274, 1999.

40. Brewis N, Phelan A, Webb J, Drew J, Elliott G and O'Hare P. Evaluation of VP22 spread in tissue culture. J Virol 74: 1051-1056, 2000.

41. Aints A, Dilber MS and Smith CI: Intercellular spread of GFP-VP22. J Gene Med 1: 275-279, 1999.

42. Bitzer M and Lauer UM: Oncolytic viruses for genetic therapy of gastrointestinal tumors. Z Gastroenterol 41: 667-674, 2003.

43. Everts B and van der Poel HG: Replication-selective oncolytic viruses in the treatment of cancer. Cancer Gene Ther 12: 141-161, 2005. 